\title{
Produção, composição da uva e teores de nitrogênio na folha e no pecíolo em videiras submetidas à adubação nitrogenada
}

\author{
Grapes yield, composition and nitrogen content in leaf and leaf petioles in grapevine with nitrogen \\ fertilization
}

\author{
Gustavo Brunetto ${ }^{\mathrm{I}^{*}}$ Cleber Luiz Bongiorno ${ }^{\text {II }}$ Jorge Luis MattiasII Mauro Deon ${ }^{\text {II }}$ \\ George Wellington de Melo ${ }^{\mathrm{III}}$ João Kaminski ${ }^{\mathrm{I}}$ Carlos Alberto Ceretta ${ }^{\mathrm{I}}$
}

- NOTA-

RESUMO

A videira tem grande importância econômica no Sul do Brasil, mas sua produtividade está associada à utilização de fertilizantes, especialmente nitrogenados, que afetam a produtividade e a composição das bagas. O seu estado nutricional pode ser avaliado pelo teor de $N$ na folha completa e no pecíolo. O trabalho objetivou avaliar como a aplicação de $N$ afeta o seu teor na folha completa e pecíolo, usados para a recomendação de $N$ à videira, e sobre a produção $e$ composição química da uva. O trabalho compreendeu dois experimentos realizados em um vinhedo comercial no município de Planalto (RS), safra 2006/07, sobre um Cambissolo. No experimento 1, videiras Bordô e no experimento 2. Couderc 13 foram submetidas à aplicação de 0, 30, $60 e$ $90 \mathrm{~kg} \mathrm{ha}^{-1}$ de $\mathrm{N}$, sendo $50 \%$ aplicado após o inicio da brotação, $25 \%$ durante a brotação e $25 \%$ durante a floração. No pleno florescimento e na mudança da cor da uva foram coletadas folhas completas (limbo+pecíolo) e pecíolos, que foram secos, moídos e preparados à análise de $N$ total. Na maturação da uva foram coletados, aleatoriamente, cinco cachos no centro e na parte externa da planta, pesados, determinado o comprimento, a largura e, em seguida, retiradas 100 bagas, pesadas e reservadas. Posteriormente, os demais cachos foram coletados e pesados para a determinação da produção de uva por planta e produção por hectare. As amostras de bagas reservadas foram trituradas e analisado o total de $N$, fósforo $(P)$ e potássio (K). Os resultados mostram de forma destacada que as amostras de folhas completas, coletadas no pleno florescimento na cultivar "Bordô" e "Couderc 13", são adequadas para avaliar a disponibilidade de $N$ na planta. A aplicação de $N$ mineral aumentou a produção de uva na cultivar "Bordô" e o total de $N$ nas bagas em ambas as cultivares.

Palavras-chave: análise de tecido, nitrogênio na baga, videira.

\section{ABSTRACT}

The vine has an economic importance in the South of Brazil, but its productivity is associated with the utilization of fertilizers, especially nitrogen $(N)$ ones, which affects the productivity and the composition of the grapes. Its nutritional state can be evaluated by the $N$ content in the foliar lamina and petiole. Two experiments were carried out in 2006/07 to evaluate the effect of the $N$ fertilization in the $N$ content of the foliar lamina and petiole, which is used for the $N$ recommendation, yield and grape composition of vine, at Haplumbrept soil in Southern Brazil, Planalto city. Bordô vines of the experiment 1 and Couderc 13 ones of the experiment 2 were submitted to applications of 0, 30, 60 and $90 \mathrm{~kg} \mathrm{ha}^{-1}$ of $\mathrm{N}$ as follow: $50 \%$ was applied after the beginning of bud burst, 25\% during bud burst and 25\% during the flowering. Samples of the foliar lamina and petiole were collected in the flowering and verasion. The samples were dried, grinded and prepared to the total $N$ analysis. By the time of the grape maturation, five bunches were collected randomly in the center and in the external part of the plant, which were weighted and submitted to length and wide measurements. Sequentially, 100 grapes were taken from these bunches, weighted and reserved. Following, the other bunches were collected and weighted, in order to determine the yield per plant and per hectare. Berries reserved were triturated and $N$-total, $P$ and $K$ were evaluated. The results clearly showed that the samples of foliar lamina collected in the flowering of

'Departamento de Solos, Centro de Ciências Rurais (CCR), Universidade Federal de Santa Maria (UFSM), CP 221, 97105-900, Santa Maria, RS, Brasil. E-mail: brunetto.gustavo@gmail.com. *Autor para correspondência.

"Centro de Ciências Agro Ambientais e de Alimentos, Universidade Comunitária Regional de Chapecó (UNOCHAPECÓ), Chapecó, SC, Brasil.

IIIEmbrapa Uva e Vinho, Bento Gonçalves, RS, Brasil. 
the 'Bordô' and 'Courdec 13' cultivars were suitable to evaluate the availability of $N$ in the plant. The $N$ fertilization had increased the yield of grape in the Bordô cultivar and the total $N$ in the grapes of both cultivars.

Key words: tissues analysis, $N$ grape, vine.

O Rio Grande do Sul (RS) é o maior produtor de uvas para a elaboração de vinhos e de sucos do Brasil e a Serra Gaúcha é a maior região vitivinícola. Nesta Região, o cultivo da videira é realizado, em geral, em solos rasos, com textura média ou argilosa, com teor médio ou alto de matéria orgânica e manutenção dos resíduos de plantas de cobertura durante todo o ano, conferindo-lhes capacidade de fornecimento de nitrogênio $(\mathrm{N})$, proporcional a mineralização da matéria orgânica lábil (BRUNETTO, 2004; BRUNETTO et al., 2006; BRUNETTO et al., 2007). Entretanto, com a ampliação de fronteiras agrícolas, áreas cultivadas com culturas anuais do Planalto do RS, foram incorporadas ao sistema de produção de uvas. Nessas, cultivares de videiras americanas, utilizadas para a produção de vinhos populares e sucos, como a "Bordô”, ou híbridas, como a "Couderc 13", passaram a ser cultivadas em solos com textura média ou argilosa e baixo ou médio teor de matéria orgânica e com pequena capacidade de suprimento de N. Assim, a aplicação de fontes minerais de $\mathrm{N}$ tornou-se uma prática de manejo recomendada, pois tem efeito no potencial de produção (BELL \& ROBSON, 1999), na composição da baga, e na acumulação de nutrientes (BRUNETTO et al., 2007; BRUNETTO, 2008). O teor de $\mathrm{N}$ na folha completa e/ou pecíolo tem sido, usados para estimar o estado nutricional da planta.

No RS e Santa Catarina (RS), a Comissão de Química e Fertilidade do Solo (CQFS-RS/SC, 2004) estabelece a dose de $\mathrm{N}$ para o ciclo seguinte das videiras a partir do teor total na folha completa ou no pecíolo, coletados na mudança da cor das bagas, estabelecendo a quantidade que utilizará para uma expectativa de produtividade. No entanto, nem sempre os órgãos amostrados são sensíveis para refletir o estado nutricional da planta e/ou a disponibilidade de $\mathrm{N}$ de solos. Além disso, esta forma de estimativa não considera a composição química da uva. Como a disponibilidade de $\mathrm{N}$ sofre variações com as condições de fertilidade do solo, com as manifestações climáticas e em fases fenológicas é necessário conhecer as implicações que terá o manejo da adubação nitrogenada na produtividade da cultivar e na qualidade de seus derivados na agroindústria. O trabalho objetivou avaliar como a aplicação de $\mathrm{N}$ afeta o seu teor na folha completa e pecíolo, usados para a recomendação de $\mathrm{N}$ à videira nos Estados do RS e SC, e sobre a produção e composição química da uva.

O trabalho compreendeu dois experimentos: experimento 1 com a cultivar "Bordô" e experimento 2 com a "Couderc 13", ambos foram conduzidos no município de Planalto (RS), região do Planalto ("Latitude 27²0’01.61” S e Longitude 5303’37.30”O), safra 2006/ 07 . As videiras dos dois experimentos foram pé franco, plantadas no ano de 2001, na densidade de 2666 plantas por hectare, espaçamento $1,5 \mathrm{~m}$ x 2,5m e conduzidas em latada. O solo dos experimentos foi um Cambissolo, e apresentava, na camada de $0-20 \mathrm{~cm}$ os seguintes atributos: argila $510 \mathrm{~g} \mathrm{~kg}^{-1}$; matéria orgânica $11 \mathrm{~g} \mathrm{~kg}^{-1}$; pH em água 5,8; Índice SMP 6,0; Ca trocável 9,2cmol $\mathrm{dm}^{-3}$; Mg trocável 2,8cmol $\mathrm{dm}^{-3}$; Al trocável 0,0 $\mathrm{cmol}_{c}$ $\mathrm{dm}^{-3}$; P disponível (Mehlich ${ }^{-1}$ ) 8,6mg dm ${ }^{-3}$ e K disponível $252 \mathrm{mg} \mathrm{dm}^{-3}$.

No experimento 1 , as videiras da cultivar “Bordô" e no experimento 2, as “Couderc 13” foram submetidas à aplicação de 0, 30, 60 e $90 \mathrm{~kg} \mathrm{ha}^{-1}$ de N de forma parcelada: 50\% no início da brotação (03/09/06); $25 \%$ na plena brotação (30/09/06) e 25\% na floração (21/10/06), parcelado conforme recomendado pela CQFS-RS/SC (2004). A fonte de $\mathrm{N}$ foi à uréia (45\% de $\mathrm{N})$, aplicada sobre a superfície do solo, sem incorporação (CQFS-RS/SC, 2004), em uma faixa de 2,0m de largura, a partir do caule da planta, acompanhando a linha de plantio. O delineamento foi de blocos ao acaso, com três repetições, e cada parcela foi formada por sete plantas (cinco avaliadas), distribuídas ao longo da linha de plantio.

No pleno florescimento e na mudança de cor das bagas (CQFS-RS/SC, 2004), foram coletadas dez folhas completas e dez pecíolos em cada planta, opostos ao primeiro cacho, contando a partir do ápice do ramo produtivo. Em seguida, foram secos em estufa com ar forçado com temperatura de $65^{\circ} \mathrm{C}$, até massa constante, moídos e preparados para a análise de $\mathrm{N}$ total, segundo metodologia proposta por TEDESCO et al. (1995). Na maturação da uva foram coletados aleatoriamente quatro cachos no centro da planta e quatro na parte externa, os quais foram pesados e determinados o comprimento e a largura. Em seguida, foi contado o número de bagas em cada cacho, coletadas bagas no topo do cacho, parte média e inferior, pesadas e reservadas, as quais compuseram uma amostra. Logo após, os cachos restantes nas plantas foram colhidos e pesados. Em seguida, as bagas de uva reservadas de cada tratamento foram trituradas 
e determinados os teores totais de $\mathrm{N}$, fósforo (P) e potássio (K), de acordo com TEDESCO et al. (1995). Os resultados obtidos foram submetidos à análise de variância e, quando os efeitos foram significativos, foram ajustadas equações de regressão, testando-se os modelos linear e quadrático pelo teste $\mathrm{F}$, escolhendose aquele com significância menor que $5 \%(P<0,05)$.

A aplicação de $\mathrm{N}$ ao longo dos ciclos vegetativo e produtivo proporcionou aumento no teor total de $\mathrm{N}$ nas folhas completas e nos pecíolos coletados no pleno florescimento e na mudança da cor da uva nas duas cultivares (Tabela 1), semelhante ao obtido por BETTIGA \& WEST (1991). Porém, convém destacar que a coleta de folhas completas no pleno florescimento foi capaz de refletir o aumento no teor de $\mathrm{N}$ no tecido com o incremento na dose de $\mathrm{N}$ aplicada. A resposta positiva às doses de $\mathrm{N}$ pode ter ocorrido pelo baixo teor de matéria orgânica do solo, que neste caso foi de $11 \mathrm{~g} \mathrm{~kg}^{-1}$, mesmo com o solo apresentando $510 \mathrm{~g} \mathrm{~kg}^{-1} \mathrm{de}$ argila e outros atributos da fertilidade em boas condições.

Na cultivar "Bordô", nota-se que houve um aumento no comprimento e na largura dos cachos com o incremento da dose de N. Convém ressaltar que os valores de produção da cultivar "Bordô" foram muito inferiores aos normalmente obtidos em vinhedos dessa cultivar no RS, em torno de 5,0mg ha-1 (ROMBALDI et al., 2004). Por outro lado, nas videiras da cultivar “Couderc 13” a aplicação de N não alterou a produção de uva por planta, por hectare e a largura dos cachos de uva, que se manteve em patamar superior a $10 \mathrm{mg}$ ha $^{-1}$ (Tabela 2), semelhante ao obtido por BRUNETTO et al. (2007), em viníferas Cabernet Sauvignon na região da Campanha do RS e BELL \& ROBSON (1999). A aplicação de $\mathrm{N}$ proporcionou aumento na massa de 100 bagas e isso pode ser atribuído, em geral, ao aumento da relação polpa/casca da baga, o que está associada à diminuição dos valores de importantes compostos responsáveis pela coloração do mosto da uva e do seu vinho, como as antocianinas (BRUNETTO et al., 2007).

A aplicação de $\mathrm{N}$ também proporcionou aumento linear no teor de $\mathrm{N}$ total na baga, em ambas as cultivares, assim como observaram SPAYD et al. (1994) e BRUNETTO et al. (2007). Com base nos dados de N na baga, se pode inferir que o mosto derivado dessas bagas tende a apresentar maior teor de $\mathrm{N}$ e isso pode evitar a sua parada de fermentação, uma vez que o $\mathrm{N}$ causa impacto na biomassa microbiana, na taxa e no tempo de fermentação (BISSON, 1991), o que é importante para obterem-se vinhos de qualidade.

Os resultados mostram de forma destacada que as amostras de folhas completas, coletadas no pleno florescimento na cultivar "Bordô" e "Couderc 13”, são adequadas para avaliar a disponibilidade de $\mathrm{N}$ na planta. A aplicação de $\mathrm{N}$ mineral aumentou a produção de uva na cultivar "Bordô" e o total de N nas bagas em ambas as cultivares.

Tabela 1 - Nitrogênio total na folha completa e no pecíolo, no pleno florescimento e na mudança da cor das bagas, em videiras da cultivar "Bordô” e “Couderc 13”, submetidas à aplicação de nitrogênio no solo e cultivadas no Planalto Gaúcho.

\begin{tabular}{|c|c|c|c|c|}
\hline \multirow{2}{*}{ Dose } & \multicolumn{2}{|c|}{-------------Pleno florescimento------------- } & \multicolumn{2}{|c|}{----------Mudança da cor das bagas------. } \\
\hline & Folha completa & Pecíolo & Folha completa & Pecíolo \\
\hline $\mathrm{kg} \mathrm{ha}^{-1}$ de $\mathrm{N}$ & \multicolumn{4}{|c|}{$\%$} \\
\hline 0 & $1,64^{1}$ & $0,73^{2}$ & $2,45^{3}$ & $0,67^{4}$ \\
\hline 30 & 2,22 & 0,71 & 1,96 & 0,79 \\
\hline 60 & 2,45 & 0,53 & 2,16 & 1,23 \\
\hline 90 & 2,57 & 0,88 & 2,51 & 1,08 \\
\hline \multirow[t]{2}{*}{$\mathrm{CV}, \%$} & 4,40 & 3,46 & 2,93 & 4,50 \\
\hline & \multicolumn{4}{|c|}{----Experimento 2 - Couderc 13---- } \\
\hline 0 & $0,67^{5}$ & $1,23^{6}$ & $2,69^{7}$ & $1,05^{8}$ \\
\hline 30 & 2,01 & 1,05 & 2,77 & 1,10 \\
\hline 60 & 2,68 & 1,75 & 2,71 & 1,14 \\
\hline 90 & 2,51 & 1,62 & 2,57 & 1,17 \\
\hline $\mathrm{CV}, \%$ & 2,61 & 4,60 & 2,24 & 3,56 \\
\hline
\end{tabular}

${ }^{(1)} \mathrm{y}=1,767+0,0101 \mathrm{x}\left(\mathrm{r}^{2}=0,89^{*}\right) ;{ }^{(2)} \mathrm{y}=0,7645-0,0083 \mathrm{x}+0,0001 \mathrm{x}^{2}\left(\mathrm{r}^{2}=0,61^{*}\right) ;{ }^{(3)} \mathrm{y}=2,423-0,0197 \mathrm{x}+0,0002 \mathrm{x}^{2}\left(\mathrm{r}^{2}=0,93^{*}\right) ;{ }^{(4)} \mathrm{y}=$ $0,692+0,0056 \mathrm{x}\left(\mathrm{r}^{2}=0,70^{*}\right) ;{ }^{(5)} \mathrm{y}=1,039+0,0206 \mathrm{x}\left(\mathrm{r}^{2}=0,77^{*}\right) ;{ }^{(6)} \mathrm{y}=1,132+0,0062 \mathrm{x}\left(\mathrm{r}^{2}=0,54^{*}\right) ;{ }^{(7)} \mathrm{y}=2,748-0,0014 \mathrm{x}\left(\mathrm{r}^{2}=0,42^{*}\right) ;{ }^{(8)} \mathrm{y}=$ $1,015-0,0400 x\left(r^{2}=0,98^{*}\right)$.

Ciência Rural, v.38, n.9, dez, 2008. 
Tabela 2 - Produção de uva, seus componentes e totais de nitrogênio, fósforo e potássio na baga em videiras da cultivar "Bordô” e “Couderc 13”, submetidas à aplicação de nitrogênio no solo e cultivadas no Planalto Gaúcho.

\begin{tabular}{|c|c|c|c|c|c|c|c|c|}
\hline \multirow{4}{*}{$\begin{array}{l}\text { Dose } \\
\mathrm{kg} \mathrm{ha}^{-1} \text { de N }\end{array}$} & \multirow{3}{*}{\multicolumn{2}{|c|}{------Produção de uva------ }} & \multicolumn{2}{|c|}{--------Cachos--------- } & \multirow{3}{*}{ Massa de 100 Bagas } & \multicolumn{3}{|c|}{------Nutrientes na baga------ } \\
\hline & & & \multirow[b]{2}{*}{ Comprimento } & \multirow[b]{2}{*}{ Largura } & & \multirow[b]{2}{*}{$\mathrm{N}$} & \multirow[b]{2}{*}{$\mathrm{P}$} & \multirow[b]{2}{*}{$\mathrm{K}$} \\
\hline & & & & & & & & \\
\hline & kg planta ${ }^{-1}$ & $\mathrm{~kg} \mathrm{ha}^{-1}$ & \multicolumn{2}{|c|}{-----------cm----------- } & ---------g--------- & \multicolumn{3}{|c|}{ 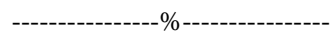 } \\
\hline & \multicolumn{8}{|c|}{---------------------------------------------------------Experimento 1 - Bordô-------------------------------------------------------- } \\
\hline 0 & $0,40^{1}$ & $1.066^{2}$ & $6,30^{3}$ & $4,43^{4}$ & $250,06^{\mathrm{ns}}$ & $0,35^{5}$ & $0,16^{\mathrm{ns}}$ & $0,30^{\mathrm{ns}}$ \\
\hline 30 & 0,80 & 2.132 & 8,15 & 5,23 & 270,39 & 0,44 & 0,16 & 0,30 \\
\hline 60 & 0,38 & 1.000 & 7,35 & 5,20 & 267,38 & 0,60 & 0,16 & 0,30 \\
\hline 90 & 0,48 & 1.279 & 8,31 & 5,46 & 257,34 & 0,54 & 0,16 & 0,27 \\
\hline $\mathrm{CV}, \%$ & 10,61 & 10,52 & 17,2 & 16,8 & 11,64 & 2,31 & 6,70 & 0,98 \\
\hline & \multicolumn{8}{|c|}{  } \\
\hline 0 & $4,73^{\mathrm{ns}}$ & $12.619^{\mathrm{ns}}$ & $13,59^{6}$ & $5,50^{\mathrm{ns}}$ & $166,46^{7}$ & $0,18^{8}$ & $0,09^{\mathrm{ns}}$ & $0,31^{\mathrm{ns}}$ \\
\hline 30 & 4,80 & 12.797 & 12,36 & 5,47 & 192,26 & 0,44 & 0,09 & 0,20 \\
\hline 60 & 3,80 & 10.131 & 13,57 & 5,87 & 196,46 & 0,34 & 0,09 & 0,31 \\
\hline 90 & 5,80 & 15.463 & 12,14 & 5,60 & 183,36 & 0,36 & 0,15 & 0,31 \\
\hline $\mathrm{CV}, \%$ & 21,75 & 21,76 & 13,5 & 16,4 & 6,16 & 4,40 & 27,53 & 25,35 \\
\hline
\end{tabular}

${ }^{\mathrm{ns}}=$ não significativo a $5 \%$ de probabilidade de erro; ${ }^{(1)} \mathrm{y}=0,467+0,0068 \mathrm{x}-0,00008 \mathrm{x}^{2}\left(\mathrm{r}^{2}=0,18^{*}\right) ;{ }^{(2)} \mathrm{y}=1246,583+18,0361 \mathrm{x}-0,21861 \mathrm{x}^{2}$ $\left(\mathrm{r}^{2}=0,18^{*}\right) ;{ }^{(3)} \mathrm{y}=6,220+0,5230 \mathrm{x}\left(\mathrm{r}^{2}=0,54^{*}\right) ;{ }^{(4)} \mathrm{y}=4,315+0,3060 \mathrm{x}\left(\mathrm{r}^{2}=0,78^{*}\right) ;{ }^{(5)} \mathrm{y}=0,373+0,0024 \mathrm{x}\left(\mathrm{r}^{2}=0,73^{*}\right) ;{ }^{(6)} \mathrm{y}=13,700-$ $0,3140 \mathrm{x}\left(\mathrm{r}^{2}=0,27^{*}\right) ;{ }^{(7)} \mathrm{y}=176,395+0,1830 \mathrm{x}\left(\mathrm{r}^{2}=0,28^{*}\right) ;{ }^{(8)} \mathrm{y}=0,264+0,0015 \mathrm{x}\left(\mathrm{r}^{2}=0,27^{*}\right)$.

\section{REFERÊNCIAS}

BELL, S.J.; ROBSON, A. Effect of nitrogen fertilization on growth, canopy density, and yield of Vitis viniferas L. cv. Cabernet Sauvignon. American Journal of Enology and Viticulture, Davis, v.50, p.351-358, 1999.

BETTIGA, L.J.; WEST, T. Tissue nitrogen in Pinot noir grapevines as affected by nitrogen fertilization timing. In: INTERNATIONAL SYMPOSIUM ON NITROGEN IN GRAPES AND WINE, 1991, Washington. Proceeding... Washington: Seattle. The American Society for Enology and Viticulture, 1991. p.221-223.

BISSON, L.F. Influence of nitrogen on yeast and fermentation of grapes. In: INTERNATIONAL SYMPOSIUM ON NITROGEN IN GRAPES AND WINE, 1991, Washington. Proceeding... Washington: Seattle. The American Society for Enology and Viticulture, 1991. p.78-89.

BRUNETTO, G. Absorção e redistribuição do nitrogênio aplicado em plantas de videira. 2004. 74f. Dissertação (Mestrado em Ciência do Solo) - Universidade Federal de Santa Maria.

BRUNETTO, G. et al. Destino do nitrogênio em videiras "Chardonnay" e "Riesilng Renano" quando aplicado no inchamento das gemas. Revista Brasileira de Fruticultura, Jaboticabal, v.28, p.497-500, 2006.

BRUNETTO, G. et al. Aplicação de nitrogênio em videiras na Campanha Gaúcha: Produtividade e características químicas do mosto da uva. Ciência Rural, Santa Maria, v.37, n.2, p.389393, 2007.

BRUNETTO, G. Nitrogênio em videira: Recuperação, acumulação e alterações na produtividade e na composição da uva. 2008. 139f. Tese (Doutorado em Ciência do Solo) - Universidade Federal de Santa Maria.

COMISSÃO DE QUÍMICA E FERTILIDADE DO SOLO - RS/ SC. Manual de adubação e calagem para os Estados do Rio Grande do Sul e de Santa Catarina. 10.ed. Porto Alegre. SBCS - Núcleo Regional Sul/UFRGS, 2004. 400p.

ROMBALDI, C.V. et al. Produtividade e qualidade de uva, cv. Bordô (IVES), sob dois sistemas de cultivo. Revista Brasileira de Agrociência, Pelotas, v.10, p.519-521, 2004.

SPAYD, S.E. et al. Nitrogen fertilization of white Riesling grapes in Washington. Must and wine composition. American Journal of Enology and Viticulture, Davis, v.45, p.34-42, 1994.

TEDESCO, M.J. et al. Análise de solo, plantas e outros materiais. Porto Alegre: UFRGS/FA/DS, 1995. 174p. 